\title{
Towards real-time cosmic-ray identification with the LOw Fre- quency ARay
}

Antonio Bonardi ${ }^{1, \star}$, Stijn Buitink ${ }^{2}$, Arthur Corstanje $^{1}$, Heino Falcke ${ }^{1,3,4}$, Brian M. Hare $^{5}$, Jörg R. Hörandel ${ }^{1,3}$, Pragati Mitra ${ }^{2}$, Katie Mulrey ${ }^{2}$, Anna Nelles ${ }^{6,7}$, Jörg P. Rachen ${ }^{1}$, Laura Rossetto ${ }^{1}$, Pim Schellart $^{8}$, Olaf Scholten ${ }^{5,9}$, Satyendra Thoudam ${ }^{10}$, Gia T.N. Trinh ${ }^{5}$, Sander ter Veen ${ }^{4}$, and Tobias Winchen ${ }^{2}$

${ }^{1}$ Department of Astrophysics/IMAPP, Radboud University, P.O. Box 9010, 6500 GL Nijmegen, The Netherlands

${ }^{2}$ Astrophysical Institute, Vrije Universiteit Brussel, Pleinlaan 2, 1050 Brussels, Belgium

${ }^{3}$ NIKHEF, Science Park Amsterdam, 1098 XG Amsterdam, The Netherlands

${ }^{4}$ Netherlands Institute of Radio Astronomy (ASTRON), Postbus 2, 7990 AA Dwingeloo, The Netherland

${ }^{5} \mathrm{KVI}$-CART, University Groningen, P.O. Box 72, 9700 AB Groningen, The Netherlands

${ }^{6}$ DESY, Platanenallee 6, 15738 Zeuthen, Germany

${ }^{7}$ Humboldt-Universität zu Berlin, Institut für Physik, Newtonstrasse 15, 12489 Berlin, Germany

${ }^{8}$ Department of Astrophysical Sciences, Princeton University, Princeton, NJ 08544, USA

${ }^{9}$ Interuniversity Institute for High-Energy, Vrije Universiteit Brussel, Pleinlaan 2, 1050 Brussels, Belgium

${ }^{10}$ Department of Physics and Electrical Engineering, Linnéuniversitetet, 35195 Växjö, Sweden

\begin{abstract}
The radio signals emitted by Extensive Air Showers have been successfully used for the last decade by LOFAR to reconstruct the properties of the primary cosmic rays. Since an effective real-time recognition system for the very short radio pulses is lacking, cosmic-ray acquisition is currently triggered by an external array of particle detector, called LORA, limiting the LOFAR collecting area to the area covered by LORA. A new algorithm for the real-time cosmic-ray detection has been developed for the LOFAR Low Band Antenna, which are sensitive between 10 and $90 \mathrm{MHz}$, and is here presented together with the latest results.
\end{abstract}

\section{Introduction}

When a cosmic ray interacts with the Earth's atmosphere, a cascade of secondary particles is originated, known as Extensive Air Shower, and a radio signal is emitted through Geomagnetic and Askaryan mechanisms. The LOw Frequency ARay (LOFAR) [1] is a multipurpose radio antenna array aimed to detect radio signals in the frequency range 10-90 $\mathrm{MHz}$ (Low Band Antennas, LBAs) and 120-240 MHz (High Band Antennas, HBAs). Radio antennas are clustered into over 50 stations, spread along central and northern Europe. The LOFAR core, where the density of stations is highest, has been used since 2011 in association with the LOfar Radboud air shower Array (LORA) [2] for detecting air showers in the energy range $10^{16}-10^{18} \mathrm{eV}$.

^e-mail: a.bonardi@astro.ru.nl 
One of the biggest challenges for the Radio detection technique is to have a real-time recognition system (also known as "radio self-trigger") for the very short radio pulses from the air showers over the overwhelming background noise (also known as "Radio Frequency Interferences", RFI). In order to bridge the gap, a self-trigger algorithm has been developed for LOFAR LBAs, which, unlike HBAs, are full-sky sensitive and, hence, best-suited for cosmic-ray detection (for more details see also [3]).

\section{The LOFAR self-trigger algorithm}

The self-trigger algorithm developed for LOFAR is based on a selection phase, where radio pulses are detected, followed by a RFI-rejection phase, where RFI pulses are discarded. Pulses passing through the RFI-rejection phase will trigger the individual LOFAR station, unless that station is part of the LOFAR core. In that case, all LOFAR core stations will be triggered.

The selection phase is based on the observation of the baseline fluctuations on each antennas. A pulse is considered as "detected" if at least half of the available antennas are above the given threshold within the waiting time $t$. The RFI rejection phase consists of a multi-parameter approach: if the pulse duration $L$ (defined as the time interval during which at least 3 antennas are above the threshold) exceeds the maximum value $L_{\max }$, or if the pulse occurs within $5 \mu$ s from the previous or following one, or if the pulse elevation angle is below 30 degrees, the pulse is rejected.

The algorithm has also been optimized to the antenna configurations which are used during LOFAR observations: "LBA inner", where the 48 innermost LBA antennas are active, and "LBA outer", where the 48 outermost are active. For the "LBA inner" configuration $t=20 \mathrm{~ns}$ and $L_{\max }=125 \mathrm{~ns}$, while for the "LBA outer" configuration $t=30 \mathrm{~ns}$ and $L_{\max }=300 \mathrm{~ns}$.

\section{Analysis and Results}

The algorithm has been tested on the LOFAR raw-data, by considering 3, 4, 5, and 10 times the baseline RMS as threshold level on each antenna. Two datasets have been used:

- LORA triggered data, consisting of the raw-data downloaded following a LORA trigger. It is best-suited for evaluating the cosmic-ray selection efficiency, but the available statistics $(\sim 8.9 \mathrm{~s}$ "LBA outer" and $1.8 \mathrm{~s}$ "LBA inner") is insufficient for estimating the RFI-rejection efficiency;

- self-triggered data w/o RFI rejection, consisting of the raw-data downloaded following a trigger from the currently installed self-trigger algorithm [4], which does not feature any RFI-rejection criterion. It is best-suited for evaluating the RFI-rejection efficiency, while the observation time (24 hours with "LBA outer" only) does not allow to evaluate the cosmic-ray detection efficiency.

The results obtained with both dataset are summarized in Table 1, where it is visible that better perfomances are achieved with "LBA outer" compared to "LBA inner" configuration, especially concerning the rejection of real cosmic-ray signal. The reason is the new algorithm has been optimized for "LBA outer" configuration, because is more often used during LOFAR astronomical observations. No cosmic-ray trigger was found in the second dataset, and 10 RFI-induced triggers (mainly due to overflying aircrafts) passed all rejection criteria. Taking into account the acquisition dead-time, the obtained RFI-trigger rate was $0.5-1$ trigger per hour.

In Fig. (1) the energy distributions of the air showers generating a trigger in at least one station (at left) and the station trigger probability as function of the station distance from the shower axis (at right) are shown for the "LBA outer" configuration (similar distributions have been obtained for "LBA inner" configuration, but are omitted because of the available space). 
Table 1. Results of the new radio self-trigger algorithm applied on the LORA triggered dataset. Note: since the trigger is evaluated for each individual station, one single shower can generate a trigger on several stations.

\begin{tabular}{|c|c|c|c|}
\hline \multicolumn{4}{|c|}{ LBA inner } \\
\hline threshold & 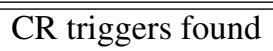 & rejected CR triggers & RFI triggers \\
\hline 3 RMS & 447 & $313(70 \%)$ & 0 \\
\hline 4 RMS & 280 & $86(31 \%)$ & 0 \\
\hline 5 RMS & 193 & $44(23 \%)$ & 0 \\
\hline 10 RMS & 62 & $22(35 \%)$ & 0 \\
\hline \multicolumn{4}{|c|}{ LBA outer } \\
\hline threshold & CR triggers found & rejected CR triggers & RFI triggers \\
\hline 3 RMS & 1520 & $182(12 \%)$ & 5 \\
\hline 4 RMS & 898 & $41 \quad(5 \%)$ & 5 \\
\hline 5 RMS & 598 & $17(3 \%)$ & 4 \\
\hline 10 RMS & 191 & $3(2 \%)$ & 2 \\
\hline
\end{tabular}
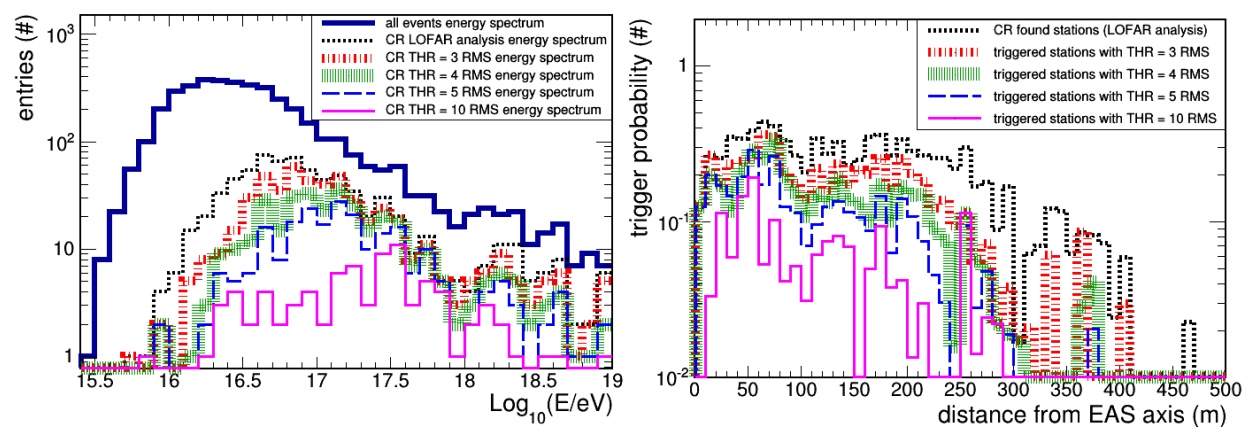

Figure 1. Left - Energy distribution of the LORA triggered dataset with "LBA outer" configuration: the whole set of showers (dark blue), the showers detected with the LOFAR off-line analysis (in black), and the showers detected with the self-trigger algorithm with threshold of 3 (red), 4 (green), 5 (blue), and 10 RMS (magenta). Right - Trigger probability for showers with energy above $2 \times 10^{7} \mathrm{eV}$ and "LBA outer" configuration vs. the station distance from the shower axis for the LOFAR offline analysis (black) and the self-trigger algorithm with threshold 3 (red), 4 (green), 5 (blue), and 10 RMS (magenta).

\section{Conclusions}

A new radio cosmic-ray self-trigger algorithm, with a good cosmic-ray detection efficiency (around $20 \%$ for $\mathrm{E}>2 \times 10^{17} \mathrm{eV}$ within $200 \mathrm{~m}$ with threshold level $4 \mathrm{RMS}$ ) and a good RFI-trigger rejection efficiency ( $<1$ event per hour), has been developed for the LOFAR observatory. The new algorithm does not require any change to LOFAR hardware, and it is planned to be installed soon.

\section{References}

[1] M.P. van Haarlem et al., Astronomy \& Astrophysics 556, 56 (2013)

[2] S. Thoudam et al., Nucl. Instrum. Meth. A 767, 339-346 (2014)

[3] A. Bonardi et al., Proceedings of the $35^{\text {th }}$ ICRC conference POS(ICRC2017)402, (2017)

[4] A. Corstanje et al., Proceedings of the $32^{\text {nd }}$ ICRC conference ID 916, 192-195 (2011) 